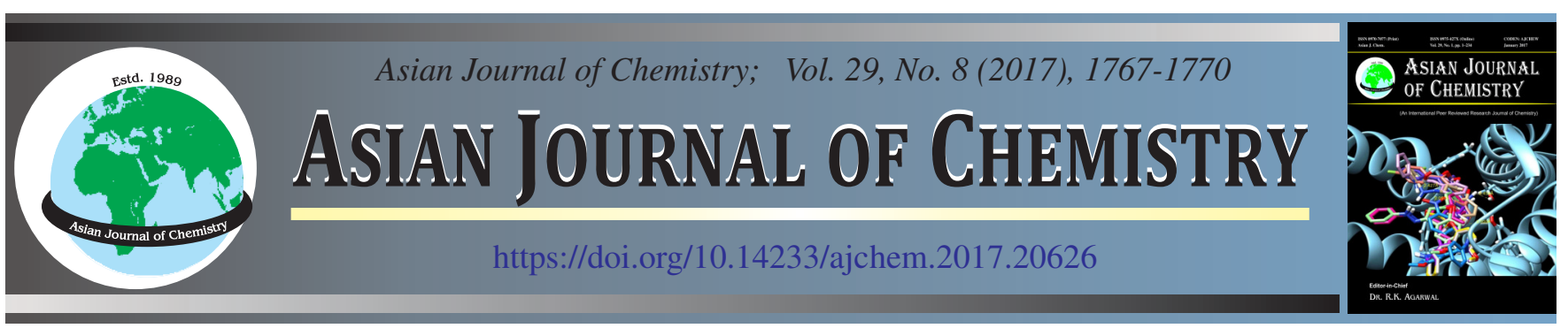

\title{
Design, Microwave Assisted Synthesis and Characterization of Substituted 1,2,4-Oxadiazole Analogues as Promising Pharmacological Agents
}

\begin{abstract}
Katharigatta N. Venugopala
Department of Biotechnology and Food Technology, Durban University of Technology, Steve Biko Campus, Durban 4001, South Africa

*Corresponding author: Fax: +27 86 2423534; Tel: +27 31 3734887; E-mail: katharigattav@ dut.ac.za

Received: 6 March 2017;

Accepted: 1 May 2017;

Published online: 12 June 2017;

AJC-18439

Microwave assisted synthesis of a series of 3,5-disubstituted-1,2,4-oxadiazole analogues (3a-j) has been achieved between 5-(chloromethyl)3 -substituted phenyl-1,2,4-oxadiazoles (2a-j) and substituted benzophenone in presence of potassium carbonate in acetone medium. The structural elucidation of the newly synthesized 1,2,4-oxadiazole analogues was established by means of FT-IR, NMR, LC-MS and elemental analysis.

Keywords: Microwave assisted synthesis, 1,2,4-Oxadiazoles, Pharmacological properties.
\end{abstract}

\section{INTRODUCTION}

The oxadiazole molecular scaffolds are known for various pharmacological activities such as antitussive, anaesthetic [1], anthelmintic [2], anti-HIV [3], antiallergic [4], anticancer [5], anticonvulsant [6], anti-inflammatory [7], antimicrobial [8], antiplatelet, antithrombotic [9], insecticidal [10], monoamine oxidase inhibition [11], muscarinic receptor agonists [12] and selective $\mathrm{H}_{3}$ receptor antagonists [13] properties.

Encouraged by these observations and in continuation of our research work on the synthesis of novel heterocyclic compounds for pharmacological properties [14-17] and for the screening of polymorphic properties in heterocyclic compounds [18-20], it was decided to incorporate benzophenone moiety on 1,2,4-oxadiazole nucleus (Scheme-I) with the support of microwave method to study the yield and total reaction time.

\section{EXPERIMENTAL}

Commercially available chemicals were obtained from Aldrich and Merck chemical company. TLC was performed on Merck 60F-254 silica gel plates with ethyl acetate and $n$-hexane (6:4) as solvent system and visualization with UV-light and iodine chamber. Reactions were monitored by thin layer chromatography (TLC). Melting points were determined on a Buchi melting point B-545 apparatus. The FT-IR spectra were recorded on a Nicolet 6700 FT-IR spectrometry. ${ }^{1} \mathrm{H}$ NMR and ${ }^{13} \mathrm{C}$ NMR spectra were recorded on Bruker AVANCE III 400 $\mathrm{MHz}$ instruments in $\mathrm{CDCl}_{3}$ as a solvent. Chemical shifts $(\delta)$ were indicated in parts per million downfield from tetramethylsilane and the coupling constants $(J)$ are recorded in Hertz. Mass spectra were recorded using LC-MS-Agilent 1100 series with MSD (Ion trap) using $0.1 \%$ aqueous trifluoroacetic acid in acetonitrile system on C18-BDS column. Elemental analysis was performed on Thermo Finnigan FLASH EA 1112 CHN analyzer. c $\log \mathrm{P}$ of the compounds was calculated using ChemBioDraw Ultra 13.0v.

Synthesis of (5-chloro-2-((3-(substituted phenyl)-1,2,4oxadiazol-5-yl)methoxy)phenyl)(phenyl)methanone (3a-j): A mixture of reaction intermediates (2a-j, 5 mmol), substituted benzophenone $(5 \mathrm{mmol})$, absolute ethanol $(25 \mathrm{~mL})$ and $\mathrm{K}_{2} \mathrm{CO}_{3}$ $(6 \mathrm{mmol})$ was exposed to microwaves in a microwave reactor for up to $10 \mathrm{~min}$. After reaction completion, the reaction medium was allowed to attain room temperature. The reaction medium was filtered and the filtrate was evaporated to dryness and the residue obtained was purified by recrystallization method. The physicochemical characteristics of the characterized title compounds $\mathbf{3 a - j}$ are summarized in Table-1 and details are as follows:

(5-Chloro-2-((3-(4-(trifluoromethyl)phenyl)-1,2,4oxadiazol-5-yl)methoxy)phenyl)(phenyl)methanone (3a): Brown crystalline compound; FT-IR $\left(\mathrm{KBr}, v_{\max }, \mathrm{cm}^{-1}\right)$ : 3064, 1655, 1594, 1482, 1451, 1261, 738. ${ }^{1} \mathrm{H}$ NMR (400 MHz, $\left.\mathrm{CDCl}_{3}\right): \delta 8.02-8.06(\mathrm{~m}, 2 \mathrm{H}), 7.82-7.86(\mathrm{~d}, J=7.20 \mathrm{~Hz}, 2 \mathrm{H})$, 7.55-7.63 (m, 1H), 7.43-7.51 (m, 4H), 7.12-7.22 (m, 2H), 7.07$7.02(\mathrm{~d}, J=8.61 \mathrm{~Hz}, 1 \mathrm{H}), 5.23\left(\mathrm{~s}, 2 \mathrm{H}, \mathrm{OCH}_{2}\right) .{ }^{13} \mathrm{C} \mathrm{NMR}(400$ $\left.\mathrm{MHz}, \mathrm{CDCl}_{3}\right): \delta 194.18,173.94,167.65,165.97,163.44$, $153.70,136.99$, 133.53, 129.81, 129.74, 129.64, 128.47, 

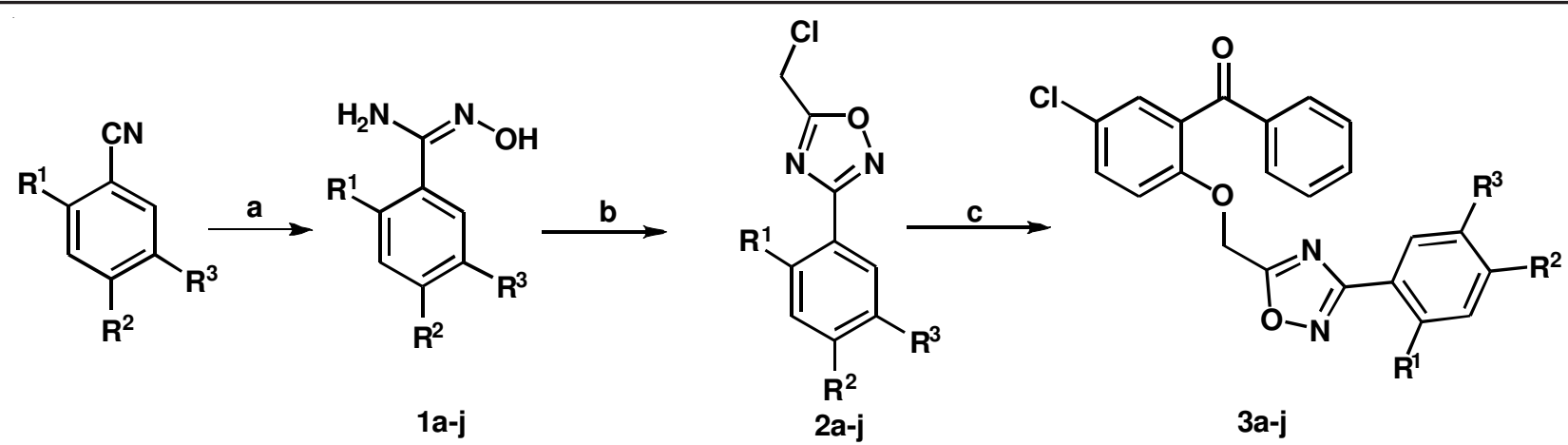

3a-j

Scheme-I: Reagents and conditions for the construction of 3,5-disubstituted 1,2,4-oxadiazoles (3a-j): (a) $\mathrm{NH}_{2} \mathrm{OH}$. $\mathrm{HCl}$, sodium carbonate, ethanol; (b) chloroacetyl chloride, N,N-diisopropylethylamine (DIEA), dichloromethene (DCM); (c) potassium carbonate, 2-hydroxy-5-chloro benzophenone, ethanol

$127.95,122.43,122.38,116.27,116.05,114.90,62.07$. LCMS (ESI, Positive): $\mathrm{m} / z=459[\mathrm{M}+\mathrm{H}]^{+}$. CHN analysis calculated for $\mathrm{C}_{23} \mathrm{H}_{14} \mathrm{~N}_{2} \mathrm{O}_{3} \mathrm{ClF}_{3}$ : C, 60.21; H, 3.08; N, 6.11; Found: C, 60.22; H, 3.05; N, 6.16 .

(5-Chloro-2-((3-(4-fluorophenyl)-1,2,4-oxadiazol-5yl)methoxy)phenyl)(phenyl)methanone (3b): Light yellow solid; FT-IR $\left(\mathrm{KBr}, \mathrm{v}_{\max }, \mathrm{cm}^{-1}\right)$ : 3063, 1651, 1592, 1482, 1451, $1264,742 .{ }^{1} \mathrm{H}$ NMR $\left(400 \mathrm{MHz}, \mathrm{CDCl}_{3}\right): \delta 8.00-8.05(\mathrm{~m}, 2 \mathrm{H})$, 7.82-7.84 (d, $J=7.24 \mathrm{~Hz}, 2 \mathrm{H}), 7.53-7.58(\mathrm{~m}, 1 \mathrm{H}), 7.41-7.48$ (m, 4H), 7.13-7.22 (m, 2H), 7.06-7.08 (d, $J=8.42 \mathrm{~Hz}, 1 \mathrm{H})$, $5.25\left(\mathrm{~s}, 2 \mathrm{H}, \mathrm{OCH}_{2}\right) .{ }^{13} \mathrm{C} \mathrm{NMR}\left(400 \mathrm{MHz}, \mathrm{CDCl}_{3}\right): \delta 194.14$, $173.97,167.61,165.98,163.42,153.70,136.99,133.53$, $131.62,131.41,129.78,129.71,129.67,128.42,127.91$, 122.42, 122.38, 116.25, 116.04, 114.89, 62.01. LC-MS (ESI, Positive): $\mathrm{m} / \mathrm{z}=410[\mathrm{M}+\mathrm{H}]^{+}$. CHN analysis calculated for $\mathrm{C}_{22} \mathrm{H}_{14} \mathrm{~N}_{2} \mathrm{O}_{3} \mathrm{ClF}$ : C, 64.64; H, 3.45; N, 6.85; Found: C, 64.61; $\mathrm{H}, 3.47$; N, 6.81 .

(5-Chloro-2-((3-(3-chloro-4-fluorophenyl)-1,2,4oxadiazol-5-yl)methoxy)phenyl)(phenyl)methanone (3c): Light yellow crystalline compound; FT-IR $\left(\mathrm{KBr}, \mathrm{v}_{\max }, \mathrm{cm}^{-1}\right)$ : 3058, 1662, 1594, 1480, 1443, 1261, 742. ${ }^{1} \mathrm{H} \mathrm{NMR}(400 \mathrm{MHz}$, $\left.\mathrm{CDCl}_{3}\right): \delta$ 8.06-8.10 (m, 1H), 7.91-7.97 (m, 1H), 7.79-7.84 $(\mathrm{m}, 2 \mathrm{H}), 7.54-7.58(\mathrm{~m}, 1 \mathrm{H}), 7.30-7.46(\mathrm{~m}, 4 \mathrm{H}), 7.21-7.28(\mathrm{~m}$, $1 \mathrm{H}), 7.04-7.09(\mathrm{~d}, J=8.42 \mathrm{~Hz}, 1 \mathrm{H}), 5.24\left(\mathrm{~s}, 2 \mathrm{H}, \mathrm{OCH}_{2}\right) .{ }^{13} \mathrm{C}$ NMR (400 MHz, $\mathrm{CDCl}_{3}$ ): $\delta$ 194.07, 174.25, 166.81, 161.27, $158.74,153.63,133.97,131.66,131.43,130.05,129.79$, $128.48,128.05,127.57,127.49,123.44,123.38,122.12$, 121.96, 117.42, 117.19, 114.92, 62.05. LC-MS: (ESI, Positive) $m / z=444[\mathrm{M}+\mathrm{H}]^{+}$. CHN analysis calculated for $\mathrm{C}_{22} \mathrm{H}_{13} \mathrm{~N}_{2} \mathrm{O}_{3} \mathrm{Cl}_{2} \mathrm{~F}$ : C, 59.61; H, 2.96; N, 6.32. Found C, 59.58; H, 3.02; N, 6.30.

(5-Chloro-2-((3-(4-fluoro-3-methylphenyl)-1,2,4oxadiazol-5-yl)methoxy)phenyl)(phenyl)methanone (3d): Light yellow solid; FT-IR (KBr, $\left.v_{\max }, \mathrm{cm}^{-1}\right)$ : 3051, 2935, 1659, $1617,1484,1450,1258,748 .{ }^{1} \mathrm{H}$ NMR $\left(400 \mathrm{MHz}, \mathrm{CDCl}_{3}\right): \delta$ 7.81-7.89 (m, 4H), 7.54-7.58 (m, 1H), 7.41-7.45 (m, 4H), 7.03$7.12(\mathrm{~m}, 2 \mathrm{H}), 5.26\left(\mathrm{~s}, 2 \mathrm{H}, \mathrm{OCH}_{2}\right), 2.32\left(\mathrm{~s}, 3 \mathrm{H}, \mathrm{CH}_{3}\right) .{ }^{13} \mathrm{C} \mathrm{NMR}$ $\left(400 \mathrm{MHz} \mathrm{CDCl}_{3}\right): \delta 194.15,173.80,167.79,164.59,162.05$, $153.72,136.98,133.51,131.41,131.33,130.88,130.83$, $129.79,128.44,127.89,127.02,126.93,125.95,125.77$, $121.98,121.94,115.83,115.62,114.87,62.05,14.52$. LC-MS (ESI, Positive): $m / z=424[\mathrm{M}+\mathrm{H}]^{+}$. CHN analysis calculated for $\mathrm{C}_{23} \mathrm{H}_{16} \mathrm{~N}_{2} \mathrm{O}_{3} \mathrm{ClF}$ : C, 65.33; H, 3.81; N, 6.63. Found C, 65.30; $\mathrm{H}, 3.89$; N, 6.58 .
(5-Chloro-2-((3-(4-fluoro-3-methoxyphenyl)-1,2,4oxadiazol-5-yl)methoxy)phenyl)(phenyl)methanone (3e): Light yellow solid; FT-IR $\left(\mathrm{KBr}, \nu_{\max }, \mathrm{cm}^{-1}\right): 3068,2981,1651$, 1630, 1498, 1441, 1253, 743. ${ }^{1} \mathrm{H}$ NMR (400 MHz, $\left.\mathrm{CDCl}_{3}\right): \delta$ 7.80-7.83 (m, 2H), 7.54-7.65 (m, 3H), 7.42-7.47 (m, 4H), 7.14$7.21(\mathrm{~m}, 1 \mathrm{H}), 7.03-7.10(\mathrm{~d}, J=8.42 \mathrm{~Hz}, 1 \mathrm{H}), 5.27\left(\mathrm{~s}, \mathrm{OCH}_{2}\right)$, $3.93\left(\mathrm{~s}, \mathrm{OCH}_{3}\right) .{ }^{13} \mathrm{C} \mathrm{NMR}\left(400 \mathrm{MHz}, \mathrm{CDCl}_{3}\right): \delta 193.97,173.89$, $167.67,167.60,156.08,153.69,152.67,148.18,147.92$, $136.95,133.47,131.58,131.30,129.68,128.42,127.87$, $122.51,122.41,120.62,120.52,116.52,116.39,114.85$, 112.19, 112.09, 62.04, 56.21. LC-MS (ESI, Positive): $\mathrm{m} / z=$ $440[\mathrm{M}+\mathrm{H}]^{+}$. CHN analysis calculated for $\mathrm{C}_{23} \mathrm{H}_{16} \mathrm{~N}_{2} \mathrm{O}_{4} \mathrm{ClF}: \mathrm{C}$, 62.95; H, 3.67; N, 6.38. Found: C, 62.98; H, 3.70; N, 6.35.

(5-Chloro-2-((3-(2,5-difluoro-4-methylphenyl)-1,2,4oxadiazol-5-yl)methoxy)phenyl)(phenyl)methanone (3f): Yellow crystalline compound; FT-IR $\left(\mathrm{KBr}, \mathrm{v}_{\max }, \mathrm{cm}^{-1}\right)$ : 3055, 2938, 1659, 1598, 1481, 1459, 1260, 743. ${ }^{1} \mathrm{H}$ NMR (400 MHz, $\left.\mathrm{CDCl}_{3}\right): \delta 7.83-7.89(\mathrm{~d}, J=7.20 \mathrm{~Hz}, 2 \mathrm{H}), 7.55-7.68(\mathrm{~m}, 2 \mathrm{H})$, 7.44-7.49 (m, 4H), 7.04-7.12 (m, 2H), 5.29 (s, 2H, $\left.\mathrm{OCH}_{2}\right)$, $2.33\left(\mathrm{~s}, 3 \mathrm{H}, \mathrm{CH}_{3}\right) .{ }^{13} \mathrm{C} \mathrm{NMR}\left(400 \mathrm{MHz}, \mathrm{CDCl}_{3}\right): \delta$ 194.11, $173.58,164.73,164.69,164.65,164.61,158.28,158.23$, $157.59,157.54,155.88,155.83,155.08,154.88,153.61$, $136.98,133.57,131.67,131.38,130.87,130.81,130.69$, $130.60,129.79,128.49,127.97,119.32,119.28,119.11$, $119.02,116.37,116.33,116.09,116.01,114.76,112.83$, 112.78, 112.72, 112.63, 61.86, 14.92, 14.81. LC-MS (ESI, Positive): $m / z=442[\mathrm{M}+\mathrm{H}]^{+}$. CHN analysis calculated for $\mathrm{C}_{23} \mathrm{H}_{15} \mathrm{~N}_{2} \mathrm{O}_{3} \mathrm{ClF}_{2}$ : C, 62.67; H, 3.43; N, 6.35. Found: C, 62.62; $\mathrm{H}, 3.51$; N, 6.39 .

(5-Chloro-2-((3-(4-iodophenyl)-1,2,4-oxadiazol-5yl)methoxy)phenyl)(phenyl)methanone (3g): Light brown crystalline compound; FT-IR $\left(\mathrm{KBr}, \mathrm{v}_{\max }, \mathrm{cm}^{-1}\right)$ : 3056, 1650, 1591, 1476, 1452, 1268, 747, 521. ${ }^{1} \mathrm{H}$ NMR (400 MHz, $\left.\mathrm{CDCl}_{3}\right): \delta 7.76-7.85(\mathrm{~m}, 6 \mathrm{H}), 7.55-7.58(\mathrm{~m}, 1 \mathrm{H}), 7.42-7.48$ (m, 4H), 7.05-7.08 (d, J=8.42 Hz, 1H), $5.22\left(\mathrm{~s}, 2 \mathrm{H}, \mathrm{OCH}_{2}\right)$. ${ }^{13} \mathrm{C}$ NMR $\left(400 \mathrm{MHz}, \mathrm{CDCl}_{3}\right): \delta 194.11,174.12,167.94$, $153.66,138.22,136.95,133.57,131.62,131.43,129.81$, $128.96,128.47,128.06,125.63,114.94,98.33,62.05$. LCMS (ESI, Positive): $m / z=518[\mathrm{M}+\mathrm{H}]^{+}$. CHN analysis calculated for $\mathrm{C}_{22} \mathrm{H}_{14} \mathrm{~N}_{2} \mathrm{O}_{3} \mathrm{ClI}$ : C, 51.14; H, 2.73; N, 5.42. Found: C, 51.17; H, 2.71; N, 5.49.

(5-Chloro-2-((3-phenyl-1,2,4-oxadiazol-5-yl)methoxy)phenyl)(phenyl)methanone (3h): White amorphous compound; 
FT-IR $\left(\mathrm{KBr}, \mathrm{v}_{\max }, \mathrm{cm}^{-1}\right): 3061,1655,1591,1484,1451,1247$, 738. ${ }^{1} \mathrm{H}$ NMR $\left(400 \mathrm{MHz}, \mathrm{CDCl}_{3}\right): \delta 7.89-8.06(\mathrm{~m}, 2 \mathrm{H}), 7.71-$ $7.78(\mathrm{~m}, 2 \mathrm{H}), 7.28-7.58(\mathrm{~m}, 8 \mathrm{H}), 7.04-7.08(\mathrm{~d}, J=8.42 \mathrm{~Hz}$, $1 \mathrm{H}), 5.23\left(\mathrm{~s}, 2 \mathrm{H}, \mathrm{OCH}_{2}\right) .{ }^{13} \mathrm{C}$ NMR $\left(400 \mathrm{MHz}, \mathrm{CDCl}_{3}\right): \delta$ $194.10,174.08,167.94,153.71,138.25,136.81,133.50$, $131.59,131.38,129.81,128.91,128.51,128.12,125.63$, 125.60, 114.93, 98.36, 62.09. LC-MS (ESI, Positive): $\mathrm{m} / \mathrm{z}=$ $392[\mathrm{M}+\mathrm{H}]^{+}$. CHN analysis calculated for $\mathrm{C}_{22} \mathrm{H}_{15} \mathrm{~N}_{2} \mathrm{O}_{3} \mathrm{Cl}: \mathrm{C}$, 67.61; H, 3.87; N, 7.17; Found: C, 67.62; H, 3.91; N, 7.22.

(5-Chloro-2-((3-(4-ethoxyphenyl)-1,2,4-oxadiazol-5yl)methoxy)phenyl)(phenyl)methanone (3i): Light yellow crystalline compound; FT-IR $\left(\mathrm{KBr}, \mathrm{v}_{\max }, \mathrm{cm}^{-1}\right)$ : 3088, 2978, 1652, 1591, 1482, 1449, 1272, 745. ${ }^{1} \mathrm{H}$ NMR (400 MHz, $\left.\mathrm{CDCl}_{3}\right): \delta$ 7.91-7.96 (m, 2H), 7.83-7.94 (m, 2H), 7.54-7.57 $(\mathrm{m}, 1 \mathrm{H}), 7.40-7.45(\mathrm{~m}, 4 \mathrm{H}), 7.03-7.06(\mathrm{~d}, J=8.42 \mathrm{~Hz}, 1 \mathrm{H})$, 6.92-6.98 (m, 2H), $5.29\left(\mathrm{~s}, 2 \mathrm{H}, \mathrm{OCH}_{2}\right), 4.05-4.14(\mathrm{q}, 2 \mathrm{H}$, $\left.\mathrm{OCH}_{2}\right), 1.42-1.48$ (t, 3H, CH 3$) .{ }^{13} \mathrm{C} \mathrm{NMR}\left(400 \mathrm{MHz}, \mathrm{CDCl}_{3}\right)$ : $\delta$ 194.07, 173.52, 168.08, 161.51, 153.61, 136.94, 133.41, $131.54,131.32,129.82,129.68,129.11,128.39,127.75$, 119.00, 114.74, 114.67, 63.60, 61.90, 14.63. LC-MS (ESI, Positive): $\mathrm{m} / \mathrm{z}=436[\mathrm{M}+\mathrm{H}]^{+}$. CHN analysis calculated for $\mathrm{C}_{24} \mathrm{H}_{19} \mathrm{~N}_{2} \mathrm{O}_{4} \mathrm{Cl}$ : C, 66.29; H, 4.40; N, 6.44. Found: C, 66.32; $\mathrm{H}, 4.39 ; \mathrm{N}, 6.47$.

(5-Chloro-2-((3-(4-isopropylphenyl)-1,2,4-oxadiazol-5yl)methoxy)phenyl)(phenyl)methanone (3j): Light yellow crystalline compound; FT-IR $\left(\mathrm{KBr}, \mathrm{v}_{\max }, \mathrm{cm}^{-1}\right)$ : 3051, 2972, 1657, 1598, 1481, 1453, 1268, 747. ${ }^{1} \mathrm{H}$ NMR (400 MHz, $\left.\mathrm{CDCl}_{3}\right): \delta$ 7.95-7.99 (m, 2H), 7.80-7.87 (m, 2H), 7.51-7.59 $(\mathrm{m}, 1 \mathrm{H}), 7.44-7.48(\mathrm{~m}, 4 \mathrm{H}), 7.34-7.36(\mathrm{~d}, J=8.16 \mathrm{~Hz}, 2 \mathrm{H})$, 7.05-7.09 (m, 1H), 5.31 (s, 2H, $\left.\mathrm{OCH}_{2}\right), 2.95-2.97$ (q, $J=6.84$ $\mathrm{Hz}, 1 \mathrm{H}), 1.27-1.31$ (d, 6H, $\left.2 \mathrm{X} \mathrm{CH}_{3}\right) .{ }^{13} \mathrm{C} \mathrm{NMR}(400 \mathrm{MHz}$, $\left.\mathrm{CDCl}_{3}\right): \delta 194.18,173.57,168.40,153.69,152.81,137.08$, $133.55,131.72,131.29,129.98,129.82,128.61,127.84$, $127.61,127.09,123.64,114.83,62.00,34.26,23.71$. LC-MS
(ESI, Positive): $\mathrm{m} / \mathrm{z}=434[\mathrm{M}+\mathrm{H}]^{+}$. $\mathrm{CHN}$ analysis calculated for $\mathrm{C}_{25} \mathrm{H}_{21} \mathrm{~N}_{2} \mathrm{O}_{3} \mathrm{Cl}$ : C, 69.36; H, 4.89; N, 6.47. Found: C, 69.42; $\mathrm{H}, 4.99 ; \mathrm{N}, 6.51$.

\section{RESULTS AND DISCUSSION}

Synthetic route for the formation of 1,2,4-oxadiazole analogues (3a-j) by microwave method is depicted in Scheme-I. The title compounds 3a-j were synthesized from substituted benzophenone and reaction intermediates 5-(chloromethyl)3-substituted phenyl-1,2,4-oxadiazoles (2a-j) in absolute ethanol medium. Intermediates $\mathbf{2} \mathbf{a}-\mathbf{j}$ were synthesized from equimolar ratios of $N$ '-hydroxybenzimidamides (1a-j) and chloroacetyl chloride in presence of DIPEA in dichloro methanol medium. The synthesis of $N^{\prime}$-hydroxybenzimidamides (1a-j) was accomplished from the corresponding hydroxylamine hydrochloride and substituted benzonitriles in presence of sodium carbonate in methanol medium. The reaction intermediates $\mathbf{1 a - j}$ and $\mathbf{2 a -}$ j were synthesized and characterized by FT-IR, ${ }^{1} \mathrm{H}$ NMR, LCMS and the characterization details matched with our previous communication [8]. The title compounds $\mathbf{3 a - j}$ were characterized by FT-IR, NMR, LC-MS and CHN analysis. The yields of intermediates 1a-j and $\mathbf{2} \mathbf{a}-\mathbf{j}$ were in the range of 58-79 and $56-75 \%$, respectively. The yield of title compounds 3,5-disubstituted 1,2,4-oxadiazoles (3a-j) was $87-93 \%$ and the physicochemical constants of the title compounds are summarized in Table-1.

In the FT-IR spectra of 3,5-disubstituted 1,2,4-oxadiazole analogues $(\mathbf{3 a}-\mathbf{j})$, carbon-chlorine and carbonyl group $(\mathrm{C}=\mathrm{O})$ peaks were found in the ranges of 725-760 and 1646-1668 $\mathrm{cm}^{-1}$, respectively. In the ${ }^{1} \mathrm{H}$ NMR spectra singlet peak in the range of 5.22-5.31 corresponds to $-\mathrm{OCH}_{2}$ - group in molecular scaffolds 3a-j. In ${ }^{13} \mathrm{C}$ NMR spectra, alkyl carbon $\left(-\mathrm{CH}_{2}-\right)$ and carbonyl carbon peaks are observed in the range of $\delta 61.86-$ 62.09 and 193.97-194.18, respectively. In LC-MS spectra,

\begin{tabular}{|c|c|c|c|c|c|c|c|c|c|c|c|}
\hline \multirow[b]{2}{*}{$\begin{array}{l}\text { Compd. } \\
\text { No. }\end{array}$} & \multicolumn{10}{|c|}{$\begin{array}{c}\text { TABLE-1 } \\
\text { PHYSICO-CHEMICAL CHARACTERISTICS OF 3,5-DISUBSTITUTED 1,2,4-OXADIAZOLES (3a-j) }\end{array}$} & \multirow[b]{2}{*}{$\mathrm{c} \log P^{\mathrm{b}}$} \\
\hline & $\mathrm{R}^{1}$ & $\mathrm{R}^{2}$ & $\mathrm{R}^{3}$ & m.f. & m.w. & $\begin{array}{c}\text { Reaction } \\
\text { time } \\
\text { (min) }\end{array}$ & $\begin{array}{l}\text { Yield } \\
(\%)\end{array}$ & $\begin{array}{c}\text { m.p. } \\
\left({ }^{\circ} \mathrm{C}\right) \\
\text { reported } \\
\text { [Ref. 8] }\end{array}$ & $\begin{array}{l}\text { m.p. } \\
\left({ }^{\circ} \mathrm{C}\right) \\
\text { found }\end{array}$ & $\begin{array}{l}\text { Crystal } \\
\text { solvent }\end{array}$ & \\
\hline $3 a$ & $\mathrm{H}$ & $\mathrm{CF}_{3}$ & $\mathrm{H}$ & $\mathrm{C}_{23} \mathrm{H}_{14} \mathrm{~N}_{2} \mathrm{O}_{3} \mathrm{ClF}_{3}$ & 458 & 4 & 91 & - & $102-103$ & Ethanol & 6.2627 \\
\hline $\mathbf{3 b}$ & $\mathrm{H}$ & $\mathrm{F}$ & $\mathrm{H}$ & $\mathrm{C}_{22} \mathrm{H}_{14} \mathrm{~N}_{2} \mathrm{O}_{3} \mathrm{ClF}$ & 408 & 6 & 88 & $96-97$ & $97-98$ & Methanol & 5.5197 \\
\hline $3 c$ & $\mathrm{H}$ & $\mathrm{F}$ & $\mathrm{Cl}$ & $\mathrm{C}_{22} \mathrm{H}_{13} \mathrm{~N}_{2} \mathrm{O}_{3} \mathrm{Cl}_{2} \mathrm{~F}$ & 442 & 5 & 93 & $85-86$ & $86-87$ & Methanol & 6.2332 \\
\hline 3d & $\mathrm{H}$ & $\mathrm{F}$ & $\mathrm{CH}_{3}$ & $\mathrm{C}_{23} \mathrm{H}_{16} \mathrm{~N}_{2} \mathrm{O}_{3} \mathrm{ClF}$ & 422 & 6 & 84 & $73-74$ & $73-74$ & Ethanol & 6.0187 \\
\hline $3 e$ & $\mathrm{H}$ & $\mathrm{F}$ & $\mathrm{OCH}_{3}$ & $\mathrm{C}_{23} \mathrm{H}_{16} \mathrm{~N}_{2} \mathrm{O}_{4} \mathrm{ClF}$ & 438 & 7 & 89 & $84-85$ & $85-86$ & Ethanol & 5.4184 \\
\hline $3 f$ & $\mathrm{~F}$ & $\mathrm{CH}_{3}$ & $\mathrm{~F}$ & $\mathrm{C}_{23} \mathrm{H}_{15} \mathrm{~N}_{2} \mathrm{O}_{3} \mathrm{ClF}_{2}$ & 440 & 5 & 92 & $87-89$ & $88-89$ & Ethanol & 6.1622 \\
\hline $3 g$ & $\mathrm{H}$ & I & $\mathrm{H}$ & $\mathrm{C}_{22} \mathrm{H}_{14} \mathrm{~N}_{2} \mathrm{O}_{3} \mathrm{ClI}$ & 516 & 6 & 87 & $124-126$ & $125-126$ & Methanol & 6.4997 \\
\hline $3 \mathrm{~h}$ & $\mathrm{H}$ & $\mathrm{H}$ & $\mathrm{H}$ & $\mathrm{C}_{22} \mathrm{H}_{15} \mathrm{~N}_{2} \mathrm{O}_{3} \mathrm{Cl}$ & 390 & 8 & 83 & $108-110$ & $109-110$ & Methanol & 5.3727 \\
\hline $3 \mathbf{i}$ & $\mathrm{H}$ & $\mathrm{OC}_{2} \mathrm{H}_{5}$ & $\mathrm{H}$ & $\mathrm{C}_{24} \mathrm{H}_{19} \mathrm{~N}_{2} \mathrm{O}_{4} \mathrm{Cl}$ & 434 & 7 & 88 & $80-82$ & $81-82$ & Ethanol & 5.9437 \\
\hline $3 \mathbf{j}$ & $\mathrm{H}$ & $\mathrm{CH}\left(\mathrm{CH}_{3}\right)_{2}$ & $\mathrm{H}$ & $\mathrm{C}_{25} \mathrm{H}_{21} \mathrm{~N}_{2} \mathrm{O}_{3} \mathrm{Cl}$ & 432 & 8 & 86 & $110-112$ & 111-112 & Ethanol & 6.7997 \\
\hline
\end{tabular}


molecular ion peaks $\left(\mathrm{M}^{+1}\right)$ of the tittle compounds $\mathbf{3 a}$-j were in good agreement with proposed molecular weight. The elemental analysis results were within $\pm 0.05 \%$ of the calculated values of the 3,5-disubstituted 1,2,4-oxadiazole analogues (3a-j). The ChemBioDraw calculated partition coefficient values of the title compounds $\mathbf{3 a - j}$ were in the range of $\mathrm{p}=$ 5.3727-6.7997. Compound with aliphatic group (isopropyl) on phenyl ring which is on third place of 1,2,4-oxadiazole nucleus exhibited highest partition value of $p=6.7997$ compared to other analogoues in the series.

\section{Conclusion}

In the present investigation, synthesis of 3,5-disubstitued 1,2,4-oxadiazole molecular scaffolds was achieved by microwave method. The functional groups such as halogens and alkoxy groups on 1,2,4-oxadiazole moiety favoured good yields and were found in the range of $87-93 \%$ after purification by recrystallization method. It was confirmed from the present investigation that, microwave method favoured to enhance the yield of the product and reduce the reaction time to obtain the title compounds $\mathbf{3 a - j}$. The Title compounds are proposed to test for suitable pharmacological properties.

\section{ACKNOWLEDGEMENTS}

The author is grateful to Durban University of Technology, and National Research Foundation, South Africa for facilities and support.

\section{REFERENCES}

1. L.A. Kayukova, K.D. Praliev, A.L. Akhelova, U.S. Kemel'bekov, G.M Pichkhadze, G.S. Mukhamedzhanova, D.M. Kadyrova and S.R. Nasyrova, Pharm. Chem. J., 45, 468 (2011);

https://doi.org/10.1007/s11094-011-0657-0.

2. A. Shaikh and J. Meshram, J. Heterocycl. Chem., 53, 1176 (2016); https://doi.org/10.1002/jhet.2377.

3. F.A. Abulwerdi, M.D. Shortridge, J. Sztuba-Solinska, R. Wilson, S.F.J. Le Grice, G. Varani and J.S. Schneekloth Jr., J. Med. Chem., 59, 11148 (2016);

https://doi.org/10.1021/acs.jmedchem.6b01450.

4. D.R. Guda, S.-J. Park, M.-W. Lee, T.-J. Kim and M.E. Lee, Eur. J. Med. Chem., 62, 84 (2013); https://doi.org/10.1016/j.ejmech.2012.12.035.
5. R. Hamdy, N.I. Ziedan, S. Ali, C. Bordoni, M. El-Sadek, E. Lashin, A. Brancale, A.T. Jones and A.D. Westwell, Bioorg. Med. Chem. Lett., 27, 1037 (2017); https://doi.org/10.1016/j.bmcl.2016.12.061.

6. M. Mohammadi-Khanaposhtani, M. Shabani, M. Faizi, I. Aghaei, R. Jahani, Z. Sharafi, N. Shamsaei Zafarghandi, M. Mahdavi, T. Akbarzadeh, S. Emami, A. Shafiee and A. Foroumadi, Eur. J. Med. Chem., 112, 91 (2016); https://doi.org/10.1016/j.ejmech.2016.01.054.

7. V.B. Iyer, B. Gurupadayya, V.S. Koganti, B. Inturi and R.S. Chandan, Med. Chem. Res., 26, 190 (2017); https://doi.org/10.1007/s00044-016-1740-6.

8. N.P. Rai, V.K. Narayanaswamy, S. Shashikanth and P.N. Arunachalam, Eur. J. Med. Chem., 44, 4522 (2009); https://doi.org/10.1016/j.ejmech.2009.06.020.

9. K. Bethge, H.H. Pertz and K. Rehse, Arch. Pharm., 338, 78 (2005); https://doi.org/10.1002/ardp.200400927.

10. J. Suzuki, D. Okamura, T. Gushikawa, K. Hirai and T. Ando, J. Pestic. Sci., 36, 392 (2011); https://doi.org/10.1584/jpestics.G11-28.

11. Y. Wang, C. Zhang, B. Zhu, Q. Li and H. Zhang, Patent No. CN104016939A (2014).

12. T. M. Twose, M. L. Verdone, R. C. Copp and J. G. Farnham, Patent No. WO2012030314A1 (2012).

13. J.W. Clitherow, P. Beswick, W.J. Irving, D.I.C. Scopes, J.C. Barnes, J. Clapham, J.D. Brown, D.J. Evans and A.G. Hayes, Bioorg. Med. Chem. Lett., 6, 833 (1996); https://doi.org/10.1016/0960-894X(96)00122-9.

14. K.N. Venugopala, G.K. Rao, P.N.S. Pai and G.L. Ganesh, Orient. J. Chem., 23, 1093 (2007).

15. A. Pathak, K.N. Venugopala, A. Joshi, G.K. Rao and K. Devi, Indian J. Heterocycl. Chem., 19, 273 (2010).

16. K.N. Venugopala, D. Rao, S. Bhandary, M. Pillay, D. Chopra, B. Aldhubiab, M. Attimarad, O. Alwassil, S. Harsha and K. Mlisana, Drug Des. Devel. Ther, 10, 2681 (2016); https://doi.org/10.2147/DDDT.S109760.

17. G.B. Dharma Rao, S. Bhandary, D. Chopra, K.N. Venugopala, R.M. Gleiser, K. Kasumbwe and B. Odhav, Chem. Biol. Drug Des., (2017); https://doi.org/10.1111/cbdd.12957.

18. P. Panini, K.N. Venugopala, B. Odhav and D. Chopra, Acta Crystallogr. $B$, 70, 681 (2014); https://doi.org/10.1107/S2052520614006209.

19. P. Munshi, K.N. Venugopala, B.S. Jayashree and T.N. Guru Row, Cryst. Growth Des., 4, 1105 (2004); https://doi.org/10.1021/cg0499481.

20. K.N. Venugopala, S.K. Nayak, R.M. Gleiser, M.E. Sanchez-Borzone, D.A. Garcia and B. Odhav, Chem. Biol. Drug Des., 88, 88 (2016); https://doi.org/10.1111/cbdd.12736. 